\title{
Vulvar Dysplastic Melanocytic Nevus
}

National Cancer Institute

\section{Source}

National Cancer Institute. Vulvar Dysplastic Melanocytic Nevus. NCI Thesaurus. Code C40334.

A rare, elevated and pigmented, compound or junctional nevus that arises from the vulva. It affects women in their reproductive years and may be associated with the presence of dysplastic nevi in other anatomic areas. It is characterized by the presence of epithelioid or spindle-shaped melanocytes exhibiting nuclear pleomorphism and prominent nucleoli. 